\title{
LAS ORGANIZACIONES EN LA ADOPCIÓN DE ESTÁNDARES EN EL SUBSECTOR HORTÍCOLA DEL NOROESTE DE MÉXICO
}

\section{ORGANIZATIONS IN THE ADOPTION OF STANDARDS SUBSECTOR HORTICULTUREIN MEXICO NORTHWEST}

\author{
Antonio M. Martínez - Reina ${ }^{1}$, Belem D. Avendaño - Ruiz², Ana I. Acosta - Martínez ${ }^{3}$ \\ Recibido para evaluación: Junio 2 de 2011 - Aceptado para publicación: Junio 14 de 2011
}

\begin{abstract}
RESUMEN
La adopción de grados y estándares en los mercados de exportación se ha convertido en una práctica generalizada. No se tiene definido el rol de las organizaciones en la adopción de estándares como tampoco hay claridad de la contribución de las organizaciones, para contribuir a que el proceso sea completo. El trabajo tiene como objetivo identificar el rol de los diferentes actores en los procesos de implementación y adopción de estándares en los productores hortícolas de la región noroeste de México. Con la información de campo, mediante una estimación econométrica se formuló un modelo Logit que permite determinar la contribución de las organizaciones en el proceso de adopción de estándares. El trabajo permite concluir que las casas comerciales hacen la mayor contribución al proceso de adopción con actividades de capacitación, asistencia técnica y financiación de insumos. Las organizaciones de productores ejercen una actividad importante especialmente en la representatividad en las negociaciones y por último las instituciones públicas son las que contribuyen en menor forma con el proceso de adopción.
\end{abstract}

Palabras Clave: mercados, calidad, normas, regulaciones.

\begin{abstract}
The adoption of grades and standards in export markets has become widespread. Has been not defined the role of the organizations in the adoption of standards and there is not clarity of the contribution of the organizations to complete the process. The objective of this work was to identify the role of different actors in the processes of implementation and adoption of standards in horticultural producers in the northwestern region of Mexico. With the field information through an econometric estimate, a Logit model was developed that allows determining the contribution of organizations in the process of adopting standards. The work concludes that
\end{abstract}

${ }^{1}$ Doctor en Ciencias Económicas Investigador unidad de análisis estratégico de CORPOICA kilómetro 14 vía Mosquera Bogotá DC Colombia. Teléfono 0914227300 correo electrónico antoniomarti40@hotmail.com.

${ }^{2}$ Profesora Investigadora Facultad de Economía y Relaciones Internacionales UABC.

${ }^{3}$ Profesora Facultad de Economía y Relaciones Internacionales UABC. 
the trading houses make the biggest contribution to the adoption process with training, technical assistance and inputs financing. Producer organizations are committed in a particularly important representation in the negotiations and finally the public institutions are contributing to a lesser way with the adoption process.

Keys word: market quality, standards, regulations.

\section{INTRODUCCIÓN}

La implementación de grados y estándares en la producción de hortalizas se ha constituido en una práctica generalizada, motivado principalmente por las exigencias de los mercados. Sin embargo, no se tiene bien definido el rol de las instituciones en los procesos de adopción y las acciones por parte de los actores con el fin de lograr un proceso integral que garantice que los productos en este caso las hortalizas alcancen los estándares exigidos por el mercado.

La región Noreoeste de México comprende los estados de Sinaloa, Sonora, Baja California Sur y Baja california Norte con gran dinamismo en la producción de hortalizas con destino a la exportación. La producción de hortalizas en esta región, es una actividad económica relevante, lo cual se atribuye a factores naturales como el clima, calidad de los suelos y la cercanía al mercado de Estados Unidos de Norteamérica. Igualmente ha creado ventajas competitivas mediante la diferenciación del producto, por su calidad, a través de la aplicación de estándares y el uso de tecnologías modernas, constituyéndose como la región líder en la producción y exportación de hortalizas frescas de México.

Este trabajo tiene como objetivo estudiar el rol de las de las organizaciones en la implementación y adopción de estándares en las hortalizas, aplicados a la región noroeste de México.

Esta investigación combina elementos teóricos como los que plantea el enfoque institucionalista quienes consideran que el mercado presenta fallas y a partir de estas surgen las instituciones como respuesta a través de normas, regulaciones y estándares.

La hipótesis que subyace en esta investigación es que las instituciones a través de las normas y regulaciones, constituyen una herramienta que facilita los procesos de implementación y adopción de grados y estándares en las empresas productoras de hortalizas, aumentando la aceptación de éstas en los mercados domésticos e internacionales y por ende su competitividad.

El documento se basa en los resultados del análisis de causalidad de la adopción de estándares mediante el uso del modelo logit y las conclusiones producto de la discusión de los datos que permiten ver el rol de las organizaciones tanto públicas como privadas en los proceso de adopción de estándares.

La aplicación de grados y estándares es importante por el hecho que los países compradores especialmente los desarrollados, son cada vez más exigentes por tanto, su cumplimiento es la clave para el comercio. Además los métodos de detección y verificación 
son más eficaces y el consumidor se ha vuelto mucho más exigente (Vagneron 2006).

La importancia de los estándares se puede entender desde el punto de vista del valor económico que puedan representar dentro del mercado, si permiten una actividad organizada y ordenada, minimizan las controversias y disputas entre compradores y vendedores, facilitan las transacciones en el comercio a distancia, diferencian los productos y permite posicionarlos en el mercado al igual que permiten la generación de información para la toma de decisiones de comprar y vender (FAO 2002).

Los productores de hortalizas del Noroeste de México han alcanzado en las últimas décadas diversos estándares de competitividad y la producción, la hacen con criterios de calidad de acuerdo con las exigencias del mercado norteamericano, la organización es un factor que les ha permitido cumplir con las exigencias de este mercado con las cantidades y calidades manteniendo una oferta permanente a lo largo del año (López 2004).

Se observa que la exigencia de normas para los países en desarrollo no necesariamente son un obstáculo aunque en algunos casos pueden ser barreras para las exportaciones. El hecho de que algunos países o industrias estén utilizando normas de alta calidad y seguridad esto pone de relieve la importancia de considerar los efectos de la inocuidad de los alimentos de origen agrícola superando las limitaciones que se puedan presentar dentro de la cadena (Henson y Hensman 2004).

Los estándares exigidos por las empresas comercializadoras de hortalizas frescas hasta la fecha son de carácter voluntario solo responden al grado de exigencia de los compradores los cuales pueden ser: Buenas Prácticas Agrícolas, Buenas Prácticas de Manejo, Buenas Prácticas de Gestión (Normas ISO 9000), inocuidad de los alimentos ISO 22000, las prácticas laborales (SA 8000 y los estándares de Comercio Justo, las normas ambientales (ISO 14000 SQF 1000/2000, IFOAM, Certificados Sanitarios, Protocolo FDA Bioterrorismo, (EE.UU.), USDA Organic (producción orgánica y organismos genéticamente modificados. (Bain, 2005).

Uno de los estándares que mayor importancia ha tomado son los de inocuidad que tienen como fin producir bienes sin que causen daños a la salud humana. Igualmente, los estándares fitosanitarios deben demostrar que el proceso productivo garantiza que el producto esté libre de patógenos, plagas, baterías y daños físicos que puedan contaminar el producto. Lo anterior se complementa con los estándares de trazabilidad que consiste en un seguimiento o rastreo que se hace a lo largo de la cadena con el fin de asegurarse que la producción se hizo bajo procesos adecuados que aseguran la calidad del producto y el cumplimiento de las normas en general (Avendaño 2006 ).

Inocuidad Alimentaria: Se define como la aplicación de prácticas de producción de alimentos de origen agrícola que tengan como fin minimizar los riesgos para la salud humana de gérmenes, toxinas y residuos químicos patógenos y a la propagación de enfermedades o parásitos que puedan afectar la salud animal o vegetal para proteger a los consumidores de los peligros involuntarios (OCED, 1999).

Referente a los gustos y preferencias del 
consumidor se aprecia una creciente demanda de una calidad superior la cual involucra aspectos externos como: presentación, apariencia, uniformidad, madurez, frescura, los cuales se constituyen en los componentes principales de la decisión de compra. Por otra parte está la calidad interna la que involucra atributos como: sabor, aroma, textura, valor nutritivo, ausencia de contaminantes bióticos y abióticos los cuales son no perceptibles pero no por ello menos importantes para los consumidores (Vagneron, 2006).

\section{MATERIALES Y MÉTODOS}

La información de este artículo se obtuvo en el trabajo de Campo para la tesis de doctorado del Estudiante Antonio María Martínez Reina a través del proyecto "Estándares e Instituciones Competitividad Limitada financiado con Recursos del CONACYT bajo la dirección de la doctora Belem Dolores Avendaño Ruiz y ejecutado por la Universidad Autónoma de Baja California.

El marco geográfico para este estudio fue la Región noroeste de México que comprende los estados de: Baja California, Baja California Sur, Sinaloa y Sonora.

La información utilizada en este trabajo se originó de dos fuentes: La primera proveniente de las instituciones del sector agropecuario, organizaciones de productores y estadísticas del INEGI que constituyen la información indirecta.

El trabajo de campo constituyó la fuente de información directa e incluye desde la definición de la muestra, número de encuestas a realizar y diseño del cuestionario, aplicación del cuestionario y captura de información complementada con entrevistas a agricultores y gerentes de empresas agrícolas.

Para la definición del tamaño de la muestra se aplicó la técnica de muestreo estratificado con afijación proporcional. En este caso se tomó como variable de muestreo la región, por tanto los estratos son las cuatro regiones: Baja California, Baja California Sur, Sonora y Sinaloa y de aquí se calculó el tamaño de la muestra, distribuidas proporcionalmente con un intervalo de confianza de $90 \%$ para un valor de 1,65 y un margen de error del 10 por ciento que da como resultado el número total de encuestas a realizar. La cual dio como resultado una muestra de 58 encuestas aplicadas a igual número de empresas agrícolas, distribuidas así: para Baja California 26 encuestas, para Baja California sur 14 encuestas, para Sinaloa 13 encuestas y cinco para el estado de Sonora.

\section{Diseño del Modelo Econométrico}

Para el análisis del rol de las instituciones en el proceso de adopción de estándares, se planteó una relación de variables donde la variable dependiente se define como el porcentaje o la probabilidad de aplicar estándares por parte de las empresas, la cual se explica por la acción de las instituciones.

Dado el carácter cualitativo de las preguntas, se establecieron variables binarias $y$ dicotómicas. Donde se incluyeron variables como: Organizaciones de productores, apoyo del gobierno, apoyo financiero, apoyos recibidos por las certificadoras, apoyos de las casas comerciales, apoyos recibidos de los centros de investigación, apoyos recibidos por las organizaciones de productores, apoyos 
recibidos por los centros de investigación. La información se agrupó en tablas de frecuencia y con la utilización del los programas econométricos Eviews y Stata se corrieron regresiones LOGIT, usando el método de máxima verosimilitud.

Los resultados sobre la probabilidad de que las empresas implementen grados y estándares a través de las variables explicatorias se pueden obtener a partir de un modelo logit también conocido como normit, que utilizó McFadden (1973). De acuerdo con Madala (1996), los modelos de elección binaria asumen que los individuos se enfrentan con una elección entre dos alternativas y la elección depende de características identificables. Madala (1996); Pindyck y Rubinfeld (2001), argumentan que dichos modelos se refieren a decisiones que involucran "deseo" y "capacidad." Consecuentemente, un modelo como el expresado contendrá variables explicativas de ambos elementos o atributos.

La forma inicial de plantear el modelo dicotómico será:

$$
y_{i}=\left\{\begin{array}{lll}
1 & \text { si } & I_{i}^{*}>0 \\
0 & \text { si } & I_{i}^{*} \leq 0
\end{array}\right.
$$

En este caso se supone que la función de distribución es una curva logística por tanto se utiliza el modelo logit donde se relacionan la variable endógena $\mathrm{Yi}$ con las variables explicativas $\mathrm{Xi}$ a través de una función de distribución (Gujarati 2006).

El modelo de probabilidad quedaría definido de la siguiente manera:
La función de verosimilitud tiene la siguiente forma:

$\mathrm{L}=\operatorname{Prob}(\mathrm{Y} 1, \ldots, \mathrm{YN})=\operatorname{Prob}(\mathrm{Y} 1), \ldots, \operatorname{Prob}(\mathrm{YN})$

Ahora, si se tiene en cuenta el hecho de que la probabilidad de elegir la segunda alternativa es igual a 1 menos la probabilidad de que se elija la primera y se utiliza el símbolo $\prod$ para representar el producto de varios factores, la función de verosimilitud se reduce a:

$L=. P_{1} \ldots \ldots . P_{\mathrm{n} 1}\left(1-P_{N}\right) \ldots \ldots\left(1-P_{N}\right)=\coprod_{i=1}^{n 1} P_{1}=\prod_{i-n 1+1}^{n 1}\left(1-P_{i}\right)=\prod_{i=1}^{n} P_{i}^{Y_{i}}(1-P)^{\left(1-\gamma_{j}\right)}$

El Modelo general planteado en este caso fue:

$Y=$ AdopCert $\frac{P i}{1-P i} \beta_{0}+\beta_{1} X_{1}+\beta_{2} X_{2}+\beta_{3} X_{3}+B_{n} X_{n}+e$

Donde:

$\mathrm{Y}=$ Adopción de Estándares

$\mathrm{X} 1, \quad \mathrm{X} 2 \quad \mathrm{X} 3 \quad . . \mathrm{Xn}=$ Variables explicatorias cualitativas (organizaciones, apoyos del gobierno, apoyos de las casa comerciales).

El resultado de este ejercicio es probar si las instituciones promueven los estándares y cuales hacen una contribución mayor al proceso de adopción de estándares por parte de las empresas productoras de hortalizas.

Construcción del Modelo Logit: Con la información obtenida en el trabajo de campo se construyó el modelo Logit, por considerar que los modelos de regresión cualitativa agrupados o de proporciones se consideran los más apropiados para medir la probabilidad de adopción de estándares por parte de las empresas productoras de hortalizas pues

$$
P\left(y_{i}=1\right)=P\left(y_{i}^{*}>0\right)=P\left(\mathbf{x}_{i}{ }^{\prime} \beta+u_{i}>0\right)=P\left(u_{i}>0-\mathbf{x}_{i}^{\prime} \beta\right)=P\left(u_{i} \leq \mathbf{x}_{i}{ }^{\prime} \beta-0\right)=F\left(\mathbf{x}_{i}^{\prime} \beta^{*}\right)
$$


permiten determinar la relación entre las probabilidades de adoptar los estándares y los efectos de las instituciones dentro del mismo proceso atendiendo a características de la producción y la estructura organizacional.
La presentación de las variables que componen el modelo se puede apreciar en la tabla 1.

La ecuación de regresión para explicar la probabilidad de estar certificadas a través las variables explicativas se muestra así:

$$
Y=\operatorname{Adop} \frac{P i}{1-P i}\left(-\left(\beta_{08}\right)+\beta_{1}\right) * \text { ORG }+\beta_{2} * \text { AGOB }+\beta_{3}^{*}(\text { APROOVED })+\mathrm{e}
$$

Tabla 1. Variables que conforman el modelo para la medición de la Adopción de Estándares en la producción de hortalizas en la Región Noroeste de México

\begin{tabular}{|c|c|}
\hline \multicolumn{2}{|c|}{ Variable Dependiente } \\
\hline Adop & $\begin{array}{l}1=\text { Si adopta estándares } \\
0=\text { De otra forma }\end{array}$ \\
\hline \multicolumn{2}{|c|}{ Variables Independientes } \\
\hline Organización & $\begin{array}{l}1=\text { Si pertenece a alguna organización de agricultores } \\
0=\text { De otra forma }\end{array}$ \\
\hline Agob & $\begin{array}{l}1=\text { Si recibe apoyos del gobierno } \\
0=\text { De otra forma }\end{array}$ \\
\hline Aprooved & $\begin{array}{l}1=\text { Si recibe apoyo de las casas comerciales } \\
0=\text { De otra forma }\end{array}$ \\
\hline
\end{tabular}

Donde:

$\mathrm{Y}=$ Adopción de estándares por parte de las empresas productoras de hortalizas de la Región Noroeste de México.

$\beta=$ Valor de los parámetros producto de la estimación del modelo Logit.

Org = Organizaciones de productores de hortalizas.

Agob $=$ Apoyos del Gobierno o entidades publicas estatales.

Aprooved $=$ apoyo de las casas comerciales o la empresas que proveen insumos.

e $=$ Termino de perturbación.
Es de anotar que habrá tantos parámetros como variables independientes haya.

De acuerdo con esta ecuación la probabilidad de explicar el proceso de adopción de estándares por parte de las empresas productoras de hortalizas puede ser explicada por la acción de las instituciones tanto públicas y privadas como también el rol que cumplen las organizaciones de productores.

La ecuación expresada en los valores de los parámetros se presenta así:

$$
Y=\operatorname{Adop} \frac{P i}{1-P i} 1-\operatorname{CNORM}(0,8310418)+2,563544 * \text { ORG }+3,096 * \text { AGOB }+11,45938 * A P R O O V E D+e
$$


Donde:

Y = Adopción de estándares por parte de las empresas productoras de hortalizas de la Región Noroeste de México.

$\beta=$ Valor de los parámetros producto de la estimación del modelo Logit.

CNORM = Intercepto no tiene significado económico.

Org = Organizaciones de productores de hortalizas.

Agob $=$ Apoyos del Gobierno o entidades publicas estatales.

Aprooved $=$ Apoyo de las casas comerciales o la empresas que proveen insumos.

e = Termino de perturbación.

Es de anotar que habrá tantos parámetros como variables independientes haya.

El análisis del rol de las instituciones en los procesos de adopción de grados y estándares por parte de las empresas productoras de hortalizas partió de los siguientes supuestos:

a) Existe una relación directa entre la adopción de estándares y las actividades que realizan las instituciones en los en las diferentes etapas del proceso productivo.

b) La adopción de estándares implica acciones conjuntas entre las instituciones y las empresas productoras de hortalizas.
Para las pruebas de significancia se tomo un margen de error del $10 \%$ por considerar que los datos se obtuvieron de campo a través de encuestas y no es de experimentos controlados por eso el margen del error permitido es del $10 \%$ para poder.

\section{RESULTADOS Y DISCUSIÓN}

El análisis del rol de las organizaciones en los procesos de adopción de estándares en la producción de hortalizas tuvo en cuenta dos tipos de análisis uno que mide la probabilidad de que las empresas productoras de hortalizas adopten estándares a través del accionar de las instituciones. Mediante el uso de un modelo Logit que relaciona las variables a través de un análisis previo de causalidad.

La ecuación producto de la estimación de parámetros se explica a continuación (ver tabla 2) el primer parámetro que corresponde a la constante con un valor de 0,8012447 indica el intercepto o punto de corte de la función.

El valor del parámetro 2,563544 que corresponde a la variable ORG que significa que cuando las empresas pertenecen a alguna organización de productores es más factible que adopte estándares dado los apoyos y la representatividad que estas le prestan al productor.

Tabla 2. Resultados de la Estimación del Modelo del Rol de las Organizaciones en los procesos de Adopción de Estándares 2010

\begin{tabular}{lccc}
\hline Variables independientes & COEFICIENTES & Z-STATIST & PROB \\
\hline Organización & 2,56 & 1,83 & 0,04 \\
Agob & 3,50 & 2,24 & 0,08 \\
Aprooved & 11,45 & 6,07 & 0,00 \\
McFadden $\mathrm{R}^{2}$ & 0,28 & & \\
\hline
\end{tabular}

Fuente: Datos de encuesta Universidad Autónoma de Baja California, México. 
La variable apoyos del gobierno AGOB, significa que si la empresa recibe apoyos del gobierno, la posibilidad de adoptar e implementar grados y estándares es mayor, el valor del parámetro de 3,5096 con signo positivo indica que cada vez que se aumenta la participación del sector publico con crédito, capacitación seguimiento y acompañamiento, es posible que las posibilidades de adoptar estándares aumenten.

La variable APROVEED que significa si las empresas se ven apoyados por las casas comerciales las posibilidades de adoptar estándares es mayor el coeficiente de 11,45938 el signo positivo muestra la relación directa entre las dos variables, lo que significa que la posibilidad de adoptar estándares aumenta cuando se intensifica la actividad de las casas comerciales en la asesoría técnica, la provisión de insumos y también con el crédito.

Es importante anotar que la variable apoyo de las instituciones financieras no resultó significativa y además el signo no es concordante con la lógica de la causalidad por cuanto su signo negativo demuestra que no aporta nada al modelo más bien causa el efecto contrario.

Lo anterior significa que las variables incluidas en el modelo que comprenden los apoyos de las instituciones tanto públicas como privadas permiten explicar la adopción de estándares por cuanto la acción de las instituciones tanto públicas como privadas contribuyen a mejorar el proceso de adopción e implementación de estándares, esto se evidencia en el valor de los parámetros en las tres variables explicatorias presenta signo positivo. Igualmente, los valores de los estadísticos indican la consistencia de las variables utilizadas en la construcción del modelo.

La información de la tabla dos muestra los resultados de regresión del modelo logístico dicotómico de la adopción de estándares por parte de las empresas productoras de hortalizas de la Región Noroeste. La R2 de McFadden es de 0,2836 (Valores del Pseudo- $R^{2}$ entre 0,2 y 0,4 se consideran indicativos de un ajuste del modelo relativamente alto (Domencich y McFadden 1975). Este valor no tiene la misma interpretación del $\mathrm{R}^{2}$ en las estimaciones por el método de mínimos cuadrados por tratarse en este caso de variables cualitativas y el método de máxima verosimilitud), indica que el modelo es adecuado para determinar la probabilidad de adoptar estándares. Los valores del $Z$ estadístico superan los valores de $z$ de la distribución de probabilidad correspondiente, considerando sus respectivos grados de libertad, por tanto se rechaza la hipótesis nula $\mathrm{HO}: \beta=0$, en este sentido se rechaza la hipótesis nula conjunta de que todos los coeficientes de regresión son cero, al 90 por ciento de confianza. Cabe anotar que en todos los casos fueron significativos a menos del 10 por ciento y por esta razón se acepta la hipótesis de que los $\beta$ son diferentes del cero.

Las variables incluidas en el modelo resultaron significativas al 90 por ciento por tratarse de investigación en campo no controlada cuando se analizaron en forma conjunta (Ver tabla 2), esto demuestra que un cambio en algunas de las variables independientes ocasionan cambios en la probabilidad de que las empresas adopten estándares en el sentido de aumentar los niveles de adopción cuando el 
accionar de las instituciones aumentan, dado los signos de los coeficientes que en todos los casos son positivos lo cual indica una relación positiva entre la adopción de estándares y el rol de las organizaciones.

Cuando se calcularon los efectos marginales (Los efectos marginales significan las contribuciones que hace cada una de las variables independientes en la explicación de la variable dependiente, se expresan en porcentaje de probabilidad que las variables explicatorias contribuyan individualmente. Se expresan en porcentaje. En este caso se trata de estimar en que porcentaje las organizaciones, los apoyos del gobierno y las casas comerciales contribuyen para que se produzca la adopción de estándares por parte de las empresas productoras de hortalizas de la región Noroest de México) o las contribuciones individuales, las tres variables resultaron significativas, se observa que recibir apoyos de las casas comerciales fueron los que mostraron una mayor contribución al aumento de la adopción de estándares con un valor de 0,47 indica que un aumento en un punto en las acciones de las casas comerciales hace aumentar en 47 por ciento la probabilidad de que las empresas adopten estándares. Lo anterior se explica por la diversidad de actividades en la producción de hortalizas pues además de promover sus productos, presentan asesoría técnica, financian insumos por tanto su importancia es alta en este proceso.

El papel de las organizaciones de productores en el proceso de adopción especialmente en la representatividad y la cooperación al momento de realizar trámites contribuye con las probabilidades de adoptar estándares con un valor de 0,14 muestra que un aumento de un punto en la acción de las organizaciones aumentan en 14 por ciento la probabilidad de que las empresas adopten estándares.

Por último los apoyos del gobierno hace una contribución individual con un valor de 0,12 la probabilidad de aumentar la adopción de estándares en las empresas productoras de hortalizas, lo que está mostrando que el sector público a través de la financiación, capacitación, seguimiento y acompañamiento contribuyen a un aumento de la adopción de estándares y el aumento de un punto en la participación del sector público ocasiona un aumento en un 12 por ciento la probabilidad de que las empresas se adopten estándares.

A través del análisis realizado a las empresas hortícolas encuestadas en la Región Noroeste de México se demuestra que estas incrementan la probabilidad de adoptar estándares y permanecer en el mercado internacional si reciben apoyo de las instituciones públicas y privadas en recursos financieros, capacitación, seguimiento y acompañamiento. Igualmente los análisis permiten inferir que de todas las instituciones que participan en el proceso de adopción estándares, las casas comerciales son las que mayor contribución hacen al proceso de adopción, sin embargo las organizaciones de productores realizan una contribución importante especialmente en capacitación, trámites, representatividad y negociaciones que hace se ubiquen en el segundo lugar.

Por último el sector publico participa activamente en con menor contribución, tal vez pueda entenderse por el hecho que las empresas ya han adquirido conciencia 
de la importancia de la implementación de estándares y el gobierno asume su rol como regulador del proceso.

\section{CONCLUSIONES}

El estudio del rol de las organizaciones en la adopción de estándares en la producción y comercialización de hortalizas en la región noroeste de México permitió entender cómo las exigencias del mercado, la aparición de contingencias como los brotes epidemiológicos y el surgimiento de nuevas oportunidades frente al mayor mercado del mundo, hizo que las empresas productoras de hortalizas enfrentaran el reto de crecer hacia fuera con su producción con calidad usando como estrategia la adopción de estándares mediante acciones conjuntas entre el sector público y el sector privado tales como la capacitación, asesoría técnica, el financiamiento, gestión para la calidad y seguimiento.

A través del análisis se demuestra que estas incrementan la probabilidad de adoptar estándares y permanecer en el mercado internacional si reciben apoyo de las instituciones públicas y privadas en recursos financieros, capacitación, seguimiento $y$ acompañamiento.

Igualmente los análisis permiten inferir que de todas las instituciones que participan en el proceso de adopción de estándares, las casas comerciales son las que mayor contribución hacen al proceso de adopción, sin embargo las organizaciones de productores realizan una contribución importante especialmente en capacitación, trámites, representatividad y negociaciones que hace se ubiquen en el segundo lugar. Por último el sector publico participa activamente en con menor contribución, tal vez pueda entenderse por el hecho que las empresas ya han adquirido conciencia de la importancia de la implementación de estándares y el gobierno asume su rol como regulador del proceso.

\section{REFERENCIAS}

Avendaño, B. 2006. Impacto de la Iniciativa de Inocuidad Alimentaria de Estados Unidos En las Exportaciones de Hortalizas Frescas del Noroeste de México. Región y sociedad, ISSN 0188-7408 18(36):7-36.

Bain, C. 2005. Third-party certification in the global agrifood system. Department of Sociology, Michigan State University, 422 Berkey Hall, East Lansing, MI 48823, USA Food Policy 30(2005):354-369.

FAO. 2002. Organización de las naciones unidas para la agricultura y la alimentación) en Httpp//www.fao.org [noviembre de 2010].

Domencich and McFadden 1975. Illustrates the conceptual flexibility and computational drawbacks of this approach. Journal of Economics.

Gujarati, D. 2006. Econometría, México, MacGraw Hill, 4.

Henson, S. and Hensman, M. 1998. Food Safety Regulation and the Firm. Understanding the Compliancen Process. Food Policy, 23(1):9-23. 
López, L. 2004. Cambios en la estructura de comercialización de las hortalizas mexicanas de exportación: el mercado detallista. Tesis de doctorado Universidad Autónoma de Sinaloa. p200.

Madala, G. 1996. Introducción a la econometría, México, Prentice-Hall 2.

McFadden, D. 1973. Conditional logit Analysis of Qualitative Choice Behavior. En P. Zarembka, Frontiers in Econometrics. Nueva York, Academic Press.

OCDE 1999. Organización para la Cooperación y el Desarrollo Económico Food Safety and Quiality, Trade Considerations Paris.
Pindyck, R. y D. Rubinfel 2001. Econometría: Modelos y pronósticos, México, McGraw Hill. Econometría, 4.

Vagneron, I. 2006. Impacts from FQ \& S regulations on social exclusion in agrichains (intro paper) En Memorias del seminario impacto de las normas internacionales sobre seguridad y calidad de los alimentos en los sistemas agroalimentarios. Buenos Aires Argentina. 